\title{
Ultraviolet Io footprint short timescale dynamics
}

\author{
B. Bonfond, ${ }^{1}$ J.-C. Gérard, ${ }^{1}$ D. Grodent, ${ }^{1}$ and J. Saur ${ }^{2}$ \\ Received 13 November 2006; revised 26 January 2007; accepted 5 February 2007; published 22 March 2007.
}

[1] The electromagnetic interaction between Io and Jupiter's magnetic field leads to single or multiple ultraviolet spots near the feet of the Io flux tube. Variations of spot numbers and brightness and of interspot distances have been observed to be linked to Io's position in its plasma torus. We have studied the evolution of the Io UV footprints with a time resolution of a few tens of seconds using the Space Telescope Imaging Spectrograph (STIS) in time-tag mode. We present evidence of systematic strong brightness variations of the main spots (up to 50\%) with a typical growth time of 1 minute. Additionally, unanticipated simultaneous fluctuations of both primary and secondary spots have also been found in the southern hemisphere. Our findings suggest that the footprint brightness is not only actively controlled by the plasma directly interacting with Io but also by the poorly constrained electron acceleration region between Io and Jupiter. Citation: Bonfond, B., J.-C. Gérard, D. Grodent, and J. Saur (2007), Ultraviolet Io footprint short timescale dynamics, Geophys. Res. Lett., 34, L06201, doi:10.1029/2006GL028765.

\section{Introduction}

[2] The presence of ultraviolet (UV) footprints shifted up to $15^{\circ}$ downstream from the estimated location of the extremities of the field lines passing through Io [Clarke et al., 1998] is a spectacular signature of the interaction between a satellite and its planet. These features provide an outstanding possibility to remotely sense Io's interaction with Jupiter's magnetosphere. Io's interaction is created by the motion of Io relative to the Io plasma torus (see reviews by Saur et al. [2004] and Thomas et al. [2004]), which perturbs the plasma and the magnetic field around Io, and which is also the root cause for the Io footprints (IFP).

[3] The first model for Io's interaction, the unipolarinductor model, was developed before the plasma torus was discovered. In this description, a steady state current loop is formed which connects Io with Jupiter's ionosphere along its magnetospheric field [e.g., Goldreich and LyndenBell, 1969]. Although Alfvén waves have been considered early for Io's interaction [Goldreich and Lynden-Bell, 1969; Goertz and Deift, 1973], their importance was firmly established only after the discovery of the dense Io plasma torus by Voyager [Neubauer, 1980; Goertz, 1980]. In this framework, Alfvén waves propagate the perturbations caused by Io towards Jupiter and form an Alfvén wing system, which is inclined with respect to Jupiter's magnetic

\footnotetext{
${ }^{1}$ Laboratoire de Physique Atmosphérique et Planétaire, Université de Liège, Liège, Belgium.

${ }^{2}$ Institut für Geophysik und Meteorologie, Universität zu Köln, Köln, Germany.
}

Copyright 2007 by the American Geophysical Union. 0094-8276/07/2006GL028765\$05.00 field in the downstream direction. Later models [e.g., Wright and Schwartz, 1989] predict substantial reflections of the Alfvén waves at the torus boundaries. Furthermore, models considering the electron finite inertia [Crary, 1997] imply the generation of electron beams towards Jupiter. Analysis of Galileo observations [Chust et al., 2005] suggests that the low frequency Alfvén waves should be reflected while high frequency, small scale waves are able to cross the torus boundaries. Io's interaction also generates a wake of slow plasma behind Io. The eventual acceleration of the wake particles through momentum transfer from the Jovian ionosphere drives an electric current along the field lines [Hill and Vasyliūnas, 2002; Delamere et al., 2003] that causes the trailing tail observed downstream of the footprints on ultraviolet and infrared images [e.g., Clarke et al., 2002; Connerney and Satoh, 2000].

[4] Observations of the IFP demonstrate that Io's interaction leads to acceleration and precipitation of $\sim 50 \mathrm{keV}$ electrons in the Jovian ionosphere [Gérard et al., 2002]. The collision of these particles with $\mathrm{H}_{2}$ molecules and $\mathrm{H}$ atoms causes optical emissions at both UV and IR wavelengths. The primary spot is frequently followed downstream by one or several secondary spots [Clarke et al., 2002; Connerney and Satoh, 2000]. Their location and the inter-spot distances appear to be consistent with Alfvén wave reflections inside the torus [Gérard et al., 2006]. The interaction between the plasma torus and Io's ionosphere also gives rise to auroral emissions at Io. Auroral emission is observed within Io's atmosphere on the sub-Jovian and the anti-Jovian sides of Io [Roesler et al., 1999; Oliversen et al., 2001]. The authors show that the intensity of this emission includes temporal variability on time scales of ca. $15 \mathrm{~min}$.

[5] The present study focuses on the footprint UV emissions and their fluctuations. An increase of the footprint brightness has been found when Io is near the center of the torus, where the plasma is denser [Gérard et al., 2006]. This result suggests that the IFP long timescale brightness variations are controlled by the strength of the Io-plasma torus interaction. In situ measurements [Franck and Paterson, 2000] and Io auroral observations [Roesler et al., 1999; Oliversen et al., 2001] have shown that the interaction between Io and the plasma torus contains a rapidly varying component, but no counterpart has been observed so far for the footprint. Here we present evidence that the IFP is also a highly dynamic process on timescales around one minute.

\section{Data Processing}

[6] Most of the previous work on the Io UV footprints used time integrated images provided by the successive cameras (e.g. FOC, WFPC2, STIS and ACS) on board the Hubble Space Telescope (HST). During the five Jovian auroral observation programs conducted with the HST/STIS camera throughout its lifetime (from 1997 to 2004), 

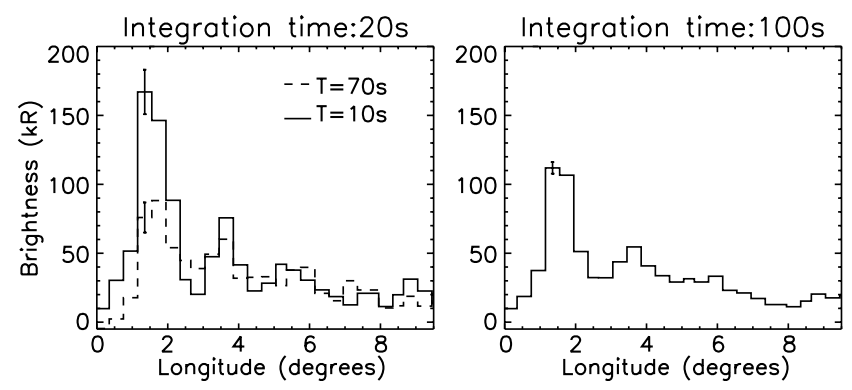

Figure 1. (left) Profiles of the northern footprint integrated over 20 seconds. The first exposure starts 10 seconds after the beginning of the time-tag sequence while the second one starts 60 seconds later. This typical time-tag sequence shows significant brightness variations of the main footprint. (right) Profile of the same northern footprint integrated over 100 seconds. The weak secondary footprint barely identifiable on 20-second exposures is now clearly distinguished. The sequence was observed the 26 February 2000 at 01:57:41 UT.

43 imaging observations ( 31 in the northern hemisphere and 12 in the southern one) were obtained in time-tag mode. In this observation mode, the positions and the arrival time of the incoming photons are registered. This specific capability of the Multi-Anode Microchannel Array (MAMA) photon counting technology has a time resolution of $125 \mu \mathrm{s}$. Each pixel of the $1024 \times 1024$ pixel detector subtends a 0.024 arcsec angle and the point spread function covers approximately a $3 \times 3$ pixels square. All the observations considered in this study were made with the SrF2 filter (central wavelength: $148 \mathrm{~nm}$, FWHM: $28 \mathrm{~nm}$ ) which rejects most of the Ly- $\alpha$ emission, largely contaminated by the geocoronal dayglow emissions. The duration of the time-tag sequences varied from 120 to 300 seconds. Most include one or two event stream interruptions due to buffer overflows. The collections of photon detection events are used to reconstruct images which may be integrated over any chosen exposure time by selecting the events in the required time range. In order to achieve an adequate balance between time-resolution and signal-to-noise ratio, we build 10-second exposure images from the time-tag datasets. The resulting sequences of images were then processed as standard STIS images, that is, dark count, flat field and geometric corrections have been performed. All time-tag observations considered here focus on the auroral morphology, but not specifically on Io footprints. Therefore, the Io footprint is not always visible nor in a favorable position. As a result, only 12 sequences in the north and 5 in the south turned out to be suitable. The Io longitudes coverage (System III) corresponding to the northern footprint observations ranges from $123^{\circ}$ to $201^{\circ}$ while the southern one spans $84^{\circ}$ to $99^{\circ}$. In order to compensate for the footprint motion during the exposure, the sub-images were shifted according to the deviation deduced from the VIP4 magnetic field model [Connerney et al., 1998]. This technique allows one to stack the footprint sub-images from the same sequence in the same reference frame. We then subtracted the Jovian background emissions in order to isolate the intrinsic IFP emissions. The footprint emission brightness as a function of longitude was obtained by extracting a 21-pixel wide stripe containing the main spots and the beginning of the tail. The brightnesses were integrated over the 21 pixels in order to obtain a linear profile. Figure 1 presents an example of a linear profile and its characteristic behaviour during a timetag sequence. Using the correction deduced from the VIP4 model, all the profiles can be plotted in the same reference frame with the $\mathrm{x}$-axis for the longitudes and the $\mathrm{y}$-axis for time to visualise the temporal evolution of the footprints. Figure 2 shows examples of the typical behaviour of aligned and color-coded profiles both for the northern and the southern hemispheres. That way, successive profiles can be summed in order to integrate the brightness over a longer time period, and therefore to increase the signal-to-noise ratio. In Figure 2, a temporal smoothing of the profiles has been performed since all the lines represent profiles with a 20 -second integration time, even if the time step between two lines is 10 seconds. The vertical alignment of the brightness peaks of the profiles in Figure 2 confirms that the VIP4 accuracy is sufficient for lining-up the profiles.

\section{Observations}

\subsection{Brightness Fluctuations}

[7] In the northern footprint brightness profiles (e.g. Figure 1), the main spot can always be clearly distinguished from the secondary ones and from the trailing tail. The second spot is usually fainter and can hardly be identified in short exposures $(\sim 10 \mathrm{~s})$ but it is revealed by increasing the integration time. Significant brightness variations of the main spot are systematically observed in all northern hemisphere datasets, ranging from $17 \%$ to $50 \%$. Figure 3 demonstrates that these fluctuations are far above the noise level and independent from background fluctuations. Moreover, a characteristic growth time of $\sim 60$ seconds can be

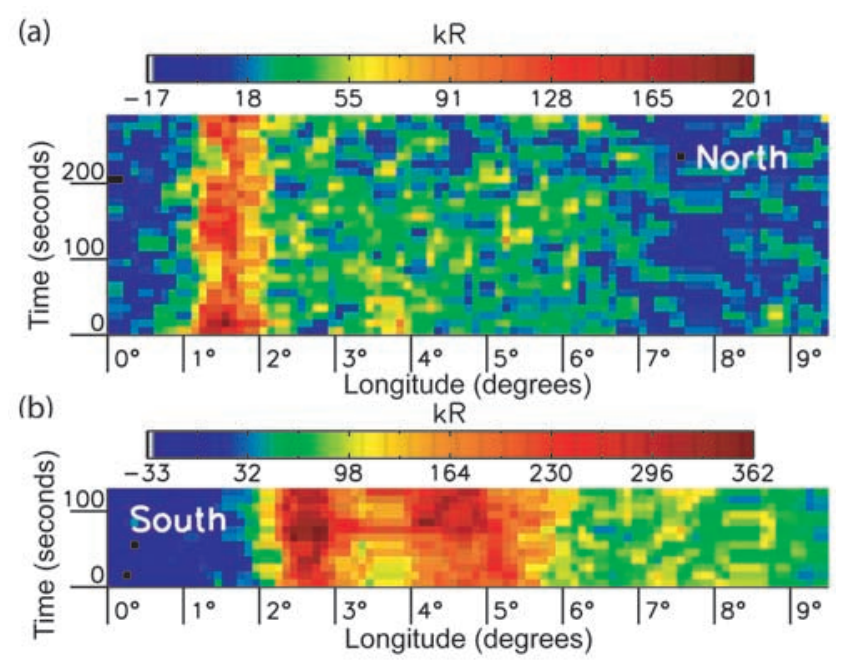

Figure 2. (a) Evolution of the profiles of the same northern footprint as in Figure 1. The horizontal axis represents the longitude (one mark is equivalent to $1^{\circ}$ ) and the vertical axis represents the time (one mark corresponds to 100 seconds). Each line is a color-coded profile with a 20 seconds exposure time. (b) Evolution of the profiles of the southern footprint sequence observed the 8 August 1999 at 12:56:47 UT. Both main and secondary footprints brightnesses increase simultaneously. 


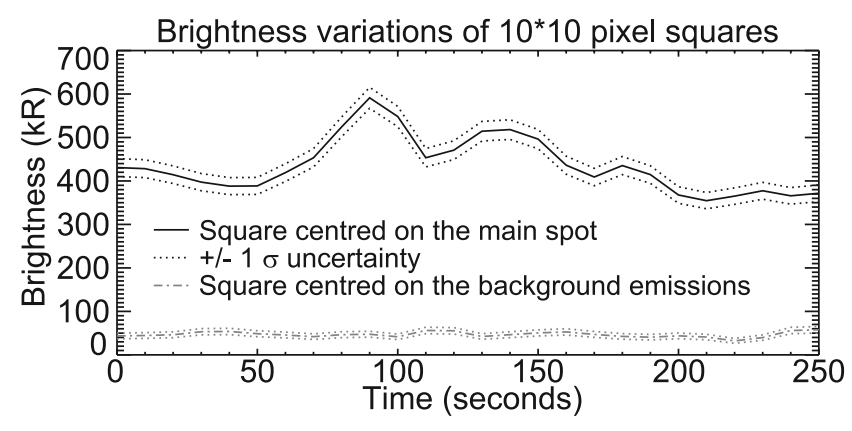

Figure 3. Light curves of $10 * 10$ pixels squares centred respectively on the main spot and on the background Jovian emissions close to the footprint. The IFP brightness fluctuations are clearly statistically significant and independent from background variations. This northern footprint sequence was observed the 16 December 2000 at 11:25:51 UT.

determined from the light curves (Figure 4a). No System III influence of the short timescale brightness variability has been identified, although the limited number of sequences does not permit any definitive conclusion. In the southern hemisphere, when a secondary spot is present, it is generally as bright or brighter than the primary spot, so that both spots can be clearly identified in the profiles. In all southern sequences, the brightness variations are on the same order of magnitude as in the north. The variations range from $25 \%$ to $36 \%$ for the primary spot and from $20 \%$ to $32 \%$ for the secondary spot.

\subsection{Simultaneous Fluctuations}

[8] In the southern hemisphere, the correlation between the brightness of the first two spots is striking (see for example Figures $4 \mathrm{~b}$ and $4 \mathrm{c}$ ). In the present dataset, the secondary spot is separated from the first one in four out of five cases. In three cases out of four, the correlation coefficients for the temporal evolution if the intensities of the primary and the secondary spot calculated for the sequences preceding the data interruptions is as high as 0.9 , largely above the significance threshold $(\sim 0.7$ at $99 \%)$. In the fourth case, no correlation is observed. These three cases occurred when Io was located in the same range of longitudes (i.e. between $84^{\circ}$ and $99^{\circ}$ ). In this sector, Io is southward from the centrifugal equator of the torus (i.e. centrifugal latitudes between $-1.2^{\circ}$ and $-2.9^{\circ}$ ), but still in its central region. Figures $4 \mathrm{~b}$ and $4 \mathrm{c}$ shows two examples of light curves of both primary and secondary southern spots. These plots suggest that in the first case, the variations of the second spot lag by $\sim 10$ seconds the variations of the first one, while in the other case the secondary spot maximum precedes the primary one. Nevertheless, considering the brightness inaccuracies and the 10 seconds time step of the samples, this slight shift of the light curves is possibly not significant.

\section{Discussion}

[9] We found significant footprint brightness variations for all observed sequences (i.e. both North and South), even though they were acquired at different times between 1999 and 2003. This provides strong evidence that the Io con- trolled auroral emissions are not in a steady state but highly dynamic. This variability may be attributed to intrinsic inhomogeneities of the interacting media (i.e. Io's ionosphere, the plasma torus, the Jovian ionosphere, etc.) or to non-linearities occurring during the propagation of the perturbation (i.e. wave breaking, phase mixing, etc.). Roesler et al. [1999] have observed fluctuations of the auroral emissions on Io with a timescale of 15 minutes and ascribed them to large-scale local variations in the plasma torus, while Oliversen et al. [2001] more precisely attributed them to electron energy flux variations in the torus. These fluctuations are one order of magnitude longer than those observed in our time-tag data. However, given the weakness of the auroral emissions on Io, the short timescale variations described here would remain indistinguishable in the auroral data for Io. Io related decametric radio emissions also show a short timescale component (S-bursts) occurring between Jupiter's surface and 0.4 Jovian radius [e.g., Ergun
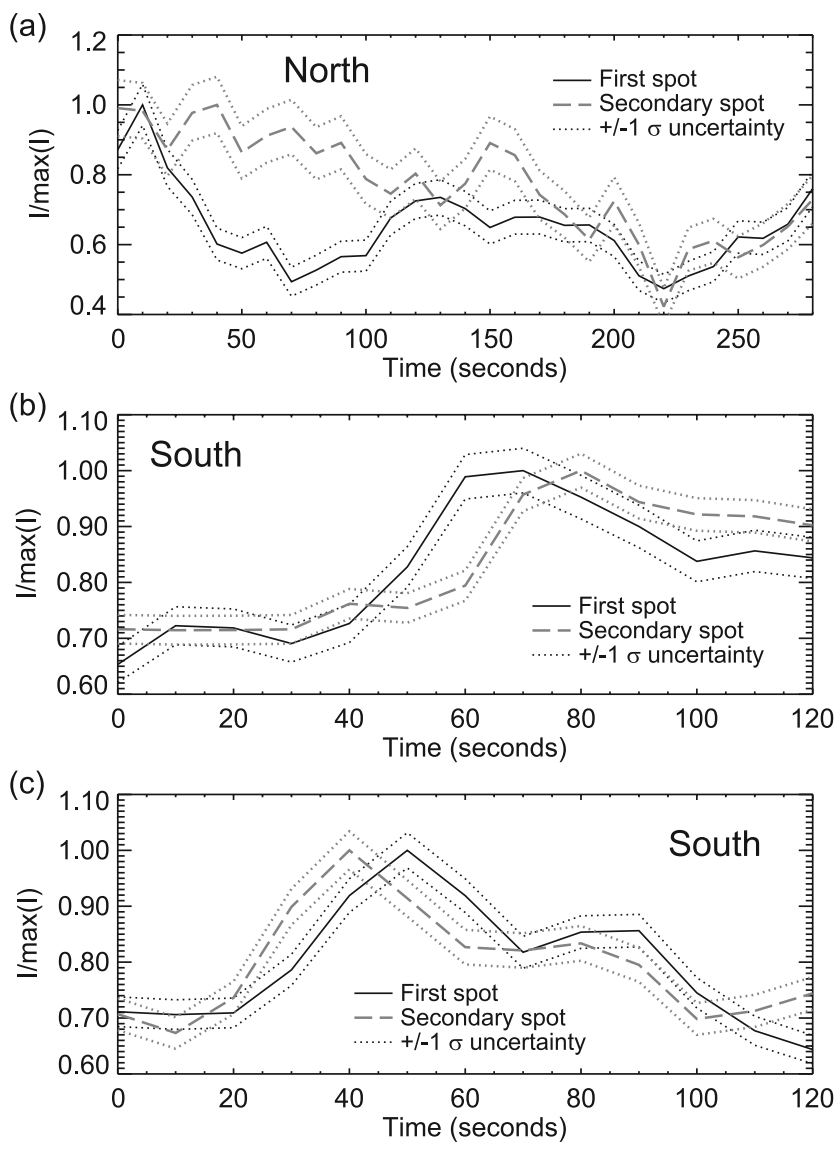

Figure 4. (a) Temporal evolution of the relative brightness of the first and the secondary spots in the profiles showed in Figure 1. The fluctuations of the first spot have a typical growth time of $\sim 1$ minute. Variations of the faint secondary spot are also present, but are not correlated to those of the main spot. (b) Temporal evolution of the relative brightness of the first and the secondary spots of a southern footprint. The sequence was observed the 8 August 1999 at 12:56:47 UT. On these light curves, the significant variations of both spots are clearly correlated. (c) Second example of time evolution of the relative brightness of the first and the secondary spots of a southern footprint observed the 12 August 2000 at 09:15:18 UT. 
et al., 2006]. However, the time scales (from 0.25 second to 0.025 second) as well as the electron energies involved ( $\sim 4 \mathrm{keV}$ with $1 \mathrm{keV}$ potential jumps) [Hess et al., 2007] can hardly be linked to the timescales $(\sim 1 \mathrm{~min})$ and the energies $(\sim 50 \mathrm{keV})$ discussed here. The simultaneous variations of the southern spots are even more intriguing. Indeed, if the brightness variations are due to a temporally varying strength of the interaction generated at Io, a delay between the primary and the secondary brightness peaks is expected. In the Alfvén wing theory, the very occurrence of the secondary spot is due to this delay, as the consequence of the longer path of the reflected perturbation. If one considers reflections inside the torus and given the Alfvén speed in the dense torus, the delay would be on the order of 13 minutes [Crary and Bagenal, 1997]. If reflections occur between the Jovian ionosphere and the outer edge of the torus, the associated delay would remain between 30 and 90 seconds. Moreover, the calculated inter-spot distance would lie between $0.14^{\circ}$ and $0.7^{\circ}$ and would be independent of Io's position in the torus, in contrast with the observations. As a consequence, even if localized fluctuations of the initial Io-plasma torus interaction may partly explain the footprint brightness variability, they provide no explanation for the simultaneous fluctuations of the southern spots. This result might imply that the acceleration mechanism leading to the electron precipitation also experiences short timescale variations. At Earth, flickering auroras are very localized spots whose brightness is probably modulated by plasma wave induced processes also occurring in the acceleration region [e.g., Sakanoi et al., 2005]. Pulsating auroras are another type of rapidly varying auroras at Earth. They appear to involve oscillations between auroral precipitation and generation of whistler waves in the equatorial plane [e.g., Nemzek et al., 1995].

\section{Conclusions}

[10] This study has unveiled a previously unexplored aspect of the Io footprint auroral emissions: their short timescale $(\sim 1$ minute) dynamics. Additionally, quasisimultaneous and highly correlated variations of primary and secondary footprint brightness suggest that the modulation is not only driven by the Io-plasma torus interaction as suggested by the long timescale brightness variations, but also by the acceleration process occurring between the torus and Jupiter. Since the acceleration mechanism is still poorly understood because of the lack of observational constraints, the amplitude of the brightness variations as well as their characteristic lengths provide essential characteristics for building future quantitative models.

[11] Acknowledgments. B. B. was supported by the PRODEX Programme managed by the ESA in collaboration with the Belgian Federal Science Policy Office and J. C. G. and D.G. by the Belgian Fund for Scientific Research (FNRS). J. S. acknowledges helpful discussion with F. M. Neubauer and S. Jacobsen. Based on observations made with the Hubble Space Telescope obtained from the ESO/ST-ECF Science Archive.

\section{References}

Chust, T., A. Roux, W. S. Kurth, D. A. Gurnett, M. G. Kivelson, and K. K. Kurana (2005), Are Io's Alfvén wings filamented? Galileo observations, Planet. Space Sci., 53, 395-412.
Clarke, J. T., et al. (1998), Hubble Space Telescope imaging of Jupiter's UV aurora during the Galileo orbiter mission, J. Geophys. Res., 103, 20,21720,236 .

Clarke, J. T., et al. (2002), Ultraviolet emissions form the magnetic footprint of Io, Ganymede and Europa on Jupiter, Nature, 415, 997-1000.

Connerney, J. E. P., and T. Satoh (2000), The H3+ ion: A remote diagnostic of the Jovian magnetosphere, Philos. Trans. R. Soc. London, Ser. A, 358, $2471-2483$

Connerney, J. E. P., M. H. Acuna, N. F. Ness, and T. Satoh (1998), New models of Jupiter's magnetic field constrained by the Io flux tube footprint, J. Geophys. Res., 103, 11,929-11,939.

Crary, F. J. (1997), On the generation of an electron beam by Io, J. Geophys. Res., 102, 37-49.

Crary, F. J., and F. Bagenal (1997), Coupling the plasma interaction at Io to Jupiter, Geophys. Res. Lett., 24, 2135-2138.

Delamere, P. A., F. Bagenal, R. Ergun, and Y.-J. Su (2003), Momentum transfer between the Io plasma wake and Jupiter's ionosphere, J. Geophys. Res., 108(A6), 1241, doi:10.1029/2002JA009530.

Ergun, R. E., Y.-J. Su, L. Andersson, F. Bagenal, P. A. Delemere, R. L. Lysak, and R. J. Strangeway (2006), $S$ bursts and the Jupiter ionospheric Alfvén resonator, J. Geophys. Res., 111, A06212, doi:10.1029/ 2005JA011253.

Franck, L. A., and W. R. Paterson (2000), Observations of plasmas in the Io torus with Galileo spacecraft, J. Geophys. Res., 105, 16,017-16,034.

Gérard, J.-C., J. Gustin, D. Grodent, P. Delamere, and J. T. Clarke (2002), Excitation of the FUV Io tail on Jupiter: Characterization of the electron precipitation, J. Geophys. Res., 107(A11), 1394, doi:10.1029/ 2002JA009410.

Gérard, J.-C., A. Saglam, D. Grodent, and J. T. Clarke (2006), Morphology of the ultraviolet Io footprint emission and its control by Io's location, J. Geophys. Res., 111, A04202, doi:10.1029/2005JA011327.

Goertz, C. K. (1980), Io's interaction with the plasma torus, J. Geophys. Res., 85, 2949-2956.

Goertz, C. K., and P. A. Deift (1973), Io's interaction with the magnetosphere, Planet. Space Sci., 21, 1399-1415.

Goldreich, P., and D. Lynden-Bell (1969), Io: A Jovian unipolar inductor, Astrophys. J., 156, 59-78.

Hess, S., P. Zarka, and F. Mottez (2007), Io-Jupiter interaction, millisecond bursts and field-aligned potentials, Planet. Space Sci., 55, 89-99, doi:10.1016/j.pss.2006.05.016.

Hill, T. W., and V. M. Vasyliūnas (2002), Jovian auroral signature of Io's corotational wake, J. Geophys. Res., 107(A12), 1464, doi:10.1029/ 2002JA009514.

Nemzek, R. J., R. Nakamura, D. N. Baker, R. D. Belian, D. J. McComas, M. F. Thomsen, and T. Yamamoto (1995), The relationship between pulsating auroras observed from the ground and energetic electrons and plasma density measured at geosynchronous orbit, J. Geophys. Res., 100(A12), 23,935-23,944.

Neubauer, F. M. (1980), Nonlinear standing Alfvén wave current system at Io: Theory, J. Geophys. Res., 85, 1171-1178.

Oliversen, R. J., F. Scherb, W. H. Smyth, M. E. Freed, R. C. Woodward Jr., M. L. Marconi, K. D. Retherford, O. L. Lupie, and J. P. Morgenthaler (2001), Sunlit Io atmospheric [O I] $6300 \AA$ A emission and the plasma torus, J. Geophys. Res., 106, 26,183-26,194.

Roesler, F. L., et al. (1999), Far-ultraviolet imaging spectroscopy of Io's atmosphere with HST/STIS, Science, 283, 353-357.

Sakanoi, K., H. Fukunishi, and Y. Kasahara (2005), A possible generation mechanism of temporal and spatial structures of flickering aurora, J. Geophys. Res., 110, A03206, doi:10.1029/2004JA010549.

Saur, J., F. M. Neubauer, J. E. P. Connerney, P. Zarka, and M. G. Kivelson (2004), Plasma interaction of Io with its plasma torus, in Jupiter: The Planet, Satellites and Magnetosphere, Cambridge Planet. Ser., vol. 1, edited by F. Bagenal, T. Dowling, and W. McKinnon, pp. 537-560, Cambridge Univ. Press, New York.

Thomas, N., F. Bagenal, T. W. Hill, and J. K. Wilson (2004), The Io neutral clouds and plasma torus, in Jupiter: The Planet, Satellites and Magnetosphere, Cambridge Planet. Ser., vol. 1, edited by F. Bagenal, T. Dowling, and W. McKinnon, pp. 561-591, Cambridge Univ. Press, New York.

Wright, A. N., and S. J. Schwartz (1989), The transmission of Alfvèn waves through the Io plasma torus, J. Geophys. Res., 94, 3749-3754.

B. Bonfond, J.-C. Gérard, and D. Grodent, Laboratoire de Physique Atmosphérique et Planétaire, Université de Liège, Liège B-4000, Belgium. (b.bonfond@ulg.ac.be)

J. Saur, Institut für Geophysik und Meteorologie, Universität zu Köln, Köln D-50923, Germany. 